\title{
Reporting of the Meditech ABPM-06 ambulatory blood pressure device validation study
}

James P Sheppard, ${ }^{1}$ Peter Lacy, ${ }^{2}$ Neil Chapman, ${ }^{3}$ Chris Clark, ${ }^{4}$ Richard McManus ${ }^{1}$

On behalf of the British and Irish Hypertension Society Blood Pressure Measurement Working Party.

${ }^{1}$ Nuffield Department of Primary Care Health Sciences, University of Oxford, UK

${ }^{2}$ Population Science \& Experimental Medicine, Institute of Cardiovascular Science, University College London, UK

${ }^{3}$ Hypertension and cardiovascular disease prevention, Imperial College Healthcare NHS Foundation Trust, UK

${ }^{4}$ Primary Care, Institute of Health Research, University of Exeter, UK

Corresponding author: James P Sheppard

Email: james.sheppard@phc.ox.ac.uk

Telephone: +44 1865617192

Address: Nuffield Department of Primary Care Health Sciences, Radcliffe Primary Care Building, Radcliffe Observatory Quarter, University of Oxford, Oxford, OX2 6GG, UK

Word count: 725 


\section{Letter to the editor}

We write as members of the British and Irish Hypertension Society Blood Pressure Monitoring Working Party regarding the article by Hermányi et al., ${ }^{1}$ published recently in Blood Pressure Monitoring. As part of our charitable work, the Society maintains the only independent list of validated monitors (available at https://bihsoc.org/bp-monitors). We maintain this list by regularly reviewing published validation studies to assess whether monitors have indeed fulfilled the strict requirements for validation studies and so should be listed on our website, regardless of the claims of the author.

In our evaluation of the article by Hermányi et al., ${ }^{1}$ we observed issues with the conduct and reporting of their validation study of the Meditech ABPM-06 ambulatory blood pressure measuring device. Specifically, the European Society of Hypertension International validation protocol 2 (ESH-IP2) ${ }^{2}$ requires that up to a maximum of four participants may have a recruitment systolic blood pressure greater than $180 \mathrm{mmHg}$ and/or less than $90 \mathrm{mmHg}$. However, in the report by Hermányi et al., ${ }^{1}$ seven participants were reported to have been recruited with systolic blood pressure above $180 \mathrm{mmHg}$ and two with systolic blood pressure below $90 \mathrm{mmHg}$. This indicates that there was over-recruitment of participants with very high systolic BP. In addition, there was subsequent under-representation of participants in the 161-180 $\mathrm{mmHg}$ range. This, we believe, constitutes a protocol deviation with regard to the entry criterion in respect of the blood pressure distribution for participants in this validation study.

In addition, the ESH-IP2 requires strict adherence to a uniform distribution of test pressures across a representative blood pressure range during the testing phase. No data were presented in the study of Hermányi and colleagues ${ }^{1}$ to confirm adherence to these study requirements. The article went on to present data evaluating the Meditech ABPM-06 ambulatory blood pressure monitor against the British Hypertension Society ${ }^{3}$ and Association for the Advancement of Medical Instrumentation (AAMI) ${ }^{4}$ protocols. Again no data were presented to confirm adherence to blood pressure distribution requirements for either protocol or the number of participants tested with each cuff for the monitor under test, as required by the AAMI protocol. Study data were presented in the form of a Bland Altman plot, but only a single plot was presented showing data from 104 participants. This yields limited information regarding the distribution and pattern of data used in the analyses for the ESH-IP2 (33 participants required) and BHS protocol (85 participants required).

Finally, a concise statement that "procedures and analysis methods of the protocols were followed precisely" was used to describe the detailed methodology followed for each protocol. This lack of detail makes it difficult to assess whether the study complied with all the requirements of the individual validation protocols: were two observers used and supervised by another? Were the observers trained? Was a period of familiarisation undertaken? What was the arm circumference of participants for each protocol and what cuff sizes were used for the monitor under test and the reference device? Were the observers blinded to one another's measurements? Were measurements taken sequentially? And what was the period between readings? We appreciate that there may be editorial constraint over the amount of space available for presenting data in journal format, especially when data from more than one protocol are acquired in validation studies. However, we believe that use of an online supplement would allow for the presentation of key information and statistics where print space is limited, allowing readers to evaluate the degree of adherence to all protocol procedures and requirements.

The Meditech ABPM-06 ambulatory blood pressure monitor may well be accurate; however, the study, as reported here, simply does not provide sufficient detail to judge whether data have been acquired according to the relevant protocols. We also raise concerns regarding a potential protocol deviation in the present report. Given the importance that members of the public, professionals and manufacturers place on claims of clinical validation, we believe that this is serious enough to warrant bringing this matter to the 
author's and editor's attention and to request publication of an erratum. We have previously raised similar concerns regarding the peer review process which has not apparently picked up these issues. ${ }^{5}$ Once again, we would urge Blood Pressure Monitoring to re-appraise this process, and ensure the standard procedures for determining the quality of the validation studies received include rigorous assessment of whether they have adhered to the validation protocols in question.

\section{Conflicts of interest}

The British and Irish Hypertension Society undertakes BP validation studies for manufacturers that are overseen by members of the Blood Pressure Measurement Working Party including the authors. 


\section{References}

1. Hermanyi Z, Pokoly B, Visolyi G, Barna I. Evaluation of Meditech ABPM-06 ambulatory blood pressure measuring device, according to the European Society of Hypertension, the British Hypertension Society and the International Organization for Standardization Protocol. Blood pressure monitoring 2019; 24(4): 208-11.

2. O'Brien E, Atkins N, Stergiou G, et al. European Society of Hypertension International Protocol revision 2010 for the validation of blood pressure measuring devices in adults. Blood pressure monitoring 2010; 15(1): 23-38.

3. O'Brien E, Petrie J, Littler W, et al. An outline of the revised British Hypertension Society protocol for the evaluation of blood pressure measuring devices. Journal of hypertension 1993; 11(6): 677-9.

4. Association for the Advancement of Medical Instrumentation. Electronic or automated sphygmomanometers ANSI/AAMI SP10-2002. 3330 Washington Boulevard, Suite 400, Arlington, VA 222014598: AAMI, 2003.

5. McManus R, Lacy P, Clark C, Chapman N, Lewis P. Reporting of blood pressure monitor validation studies. Blood pressure monitoring 2018; 23(4): 214-5. 